\title{
Erratum: Active Viscoelasticity of Odd Materials [Phys. Rev. Lett. 126, 138001 (2021)]
}

Debarghya Banerjee, Vincenzo Vitelli, Frank Jülicher, and Piotr Surówka๑

(C) (Received 1 October 2021; published 26 October 2021)

DOI: 10.1103/PhysRevLett.127.189901

In the Letter, Eq. (5) should read

$$
\bar{R}_{i j k l} \frac{d}{d t} \sigma_{k l}=-\sigma_{i j}+\eta_{i j k l} v_{k l},
$$

where, $\bar{R}_{i j k l}=\eta_{i j m n} \kappa_{m n k l}^{-1}$ is in general different from $R_{i j k l}=\kappa_{i j m n}^{-1} \eta_{m n k l}$ used in the Letter. Here, the convective derivative is defined as $d / d t=\partial / \partial t+v_{i} \partial_{i}, \sigma_{i j}$ is the stress tensor, and $v_{k l}$ is the symmetric part of the velocity gradient tensor. However, for a symmetric stress $\left(\sigma_{i j}=\sigma_{j i}\right)$, which we assume in the Letter, we obtain $\bar{R}_{i j k l}=R_{i j k l}$. Hence all following expressions, calculations, and figures presented in the Letter remain unaffected.

The authors would like to thank Professor Peter Olmsted for suggesting to clarify this point.

Published by the American Physical Society under the terms of the Creative Commons Attribution 4.0 International license. Further distribution of this work must maintain attribution to the author(s) and the published articles title, journal citation, and DOI. 